\title{
Lumbar Plexopathy Caused by Metastatic Tumor, Which Was Mistaken for Postoperative Femoral Neuropathy
}

\author{
Department of Anesthesiology and Pain Medicine, Inje University Haeundae Paik Hospital, \\ *Busan St. Mary's Medical Center, Busan, Korea \\ Ki Hwa Lee, MD, Ji Hyun Choe, MD*, Sang Eun Lee, MD, Jae Hong Park, MD, \\ Si Ra Bang, MD, Yong Han Kim, MD, and Sang Yoon Jeon, MD
}

Surgical excision was performed on a 30-years old woman with a painful mass on her left thigh. The pathologic findings on the mass indicated fibromatosis. After the operation, she complained of allodynia and spontaneous pain at the operation site and ipsilateral lower leg. We treated her based on postoperative femoral neuropathy, but symptom was aggravated. We found a large liposarcoma in her left iliopsoas muscle which compressed the lumbar plexus. In conclusion, the cause of pain was lumbar plexopathy related to a mass in the left iliopsoas muscle. Prompt diagnosis of acute neuropathic pain after an operation is important and management must be based on exact causes. (Korean J Pain 2011; 24: 226-230)

\section{Key Words:}

fibromatosis, liposarcoma, neuropathic pain.

The International Association for the Study of Pain has defined persistent postoperative pain (PPP) as chronic pain that continues for more than two months after the surgery and that cannot be explained by any clearly defined reason, such as recurrence or inflammation [1]. One of the most widely recognized causes of PPP is neuropathy resulting from either the compression, entrapment, or stretching of nerves. Risk factors of PPP include the severity of pain before and after surgery, nerve injuries, and psychosocial factors. Their impacts vary with the type and extent of surgery such as mastectomy, thoracotomy, and amputation [2].

In this case, we surgically excised a painful mass in the thigh of a patient. Following the operation, she suffered from persistent neuropathic pain that was considered postoperative femoral neuropathy and was treated as such. However, our oncology imaging accidentally discovered a metastatic tumor in her iliopsoas muscle. This discovery led to the diagnosis of lumbar plexopathy resulting from a tumor.

We report this case as well as our review of related literature to emphasize that clinicians, while treating postoperative patients with neuropathic pain, should carry out prompt and accurate diagnosis of the exact cause of postsurgical neuropathic pain and provide treatments geared specifically for that cause.

Received September 30, 2011. Revised November 15, 2011. Accepted November 15, 2011.

Correspondence to: Sang Yoon Jeon, MD

Department of Anesthesiology and Pain Medicine, Inje University Haeundae Paik Hospital, 1435, Jwa-dong, Haeundae-gu, Busan 612-862, Korea Tel: +82-51-797-0440, Fax: +82-51-797-0499, E-mail: maestro@paik.ac.kr

(a) This is an open-access article distributed under the terms of the Creative Commons Attribution Non-Commercial License (http:// creativecommons.org/licenses/by-nc/3.0/), which permits unrestricted non-commercial use, distribution, and reproduction in any medium, provided the original work is properly cited.

Copyright (c) The Korean Pain Society, 2011 


\section{CASE REPORT}

A 30-years old woman visited at our hospital, complaining of pain in her lower back, left inguinal area, and left leg. Two months before this visit, she was found to have a mass growing in her left thigh, which was causing her pain (Fig. 1). The tumor in her thigh was removed surgically. Her excisional biopsy revealed fibromatosis of the rectus femoris muscle. Following the procedure, she continued to experience pain at the operated site, as well as pain in her left inguinal region. Starting from day 3 after the surgery, the previously mentioned pains were accompanied by pain in her lower back and in the medial side of her left knee. She was asked to rate her pain using a visual analogue scale (VAS) and her rating, on average, was 100/100. She was admitted to the hospital due to the high severity of her pain. She complained of more severe pain when sitting or lying down on her back.

Physical examination found hyperesthesia and static allodynia in the left medial side of her affected leg. Both her left hip flexion (G 4/5) and left knee flexion (G 4/5) were diminished. The results of her straight leg raise test and trendelenburg test were normal. Her blood test results revealed an elevated erythrocyte sedimentation rate and C-reactive protein concentration of 56 and 4.49, respectively.

On her first day at the hospital, motor nerve conduction studies were performed. The results showed that the compound motor action potential amplitudes in her left

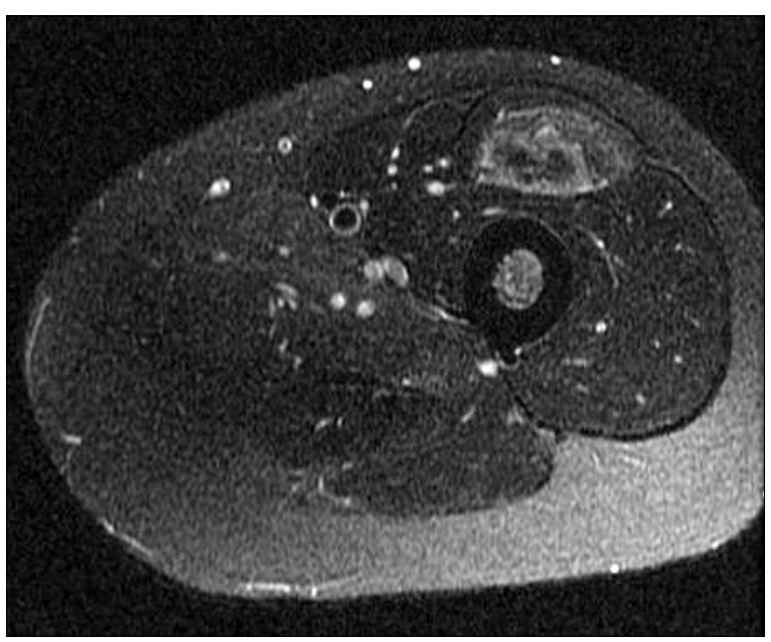

Fig. 1. Axial view (T2 WI) of thigh MRI shows intramuscular mass like lesion in left rectus femoris. femoral nerve were within the normal range but were reduced as compared with her right femoral nerve. Sensory nerve conduction tests revealed that sensory nerve action potentials were not obtained in her left saphenous nerve or in her medial femoral cutaneous nerve. Electromyography (EMG) results showed increased insertional activity in the left vastus lateralis, fibrillation, positive sharp waves $(2+)$, and diminished recruitment. Based on the results, femoral neuropathy was suspected and pregabalin $150 \mathrm{mg}$ and duloxetine $60 \mathrm{mg}$ were, accordingly, administered.

Twenty-one days into her hospitalization, the pain in her left lower leg diminished slightly, to 30/100 on her VAS rating. However, the pain in her left inguinal area and in the medial side of her left knee continued. Thus, a femoral nerve block was performed and the dosage of pregabalin was increased to $300 \mathrm{mg}$ and administered along with duloxetine $60 \mathrm{mg}$. On the patient's thirty-five days of hospitalization, the pain in her left inguinal area improved. In contrast, the pain in the medial side of her left knee worsened to $100 / 100$ on her VAS rating. Significantly reduced sensory reflex responses to cold (2/10), touch (0/10), and pinprick (0/1) were found in the medial side of her left knee. No allodynia was found. After a left L3 transforaminal epidural steroid injection, the pain in the medial side of her left knee showed an improvement based on her VAS rating of 40/100. She was, therefore, discharged from the hospital. On the thirty-six days after her first visit to the hospital, her leg pain diminished. Nevertheless, she experienced abdominal pain, nausea, and vomiting and came to the ER at the hospital to have those symptoms

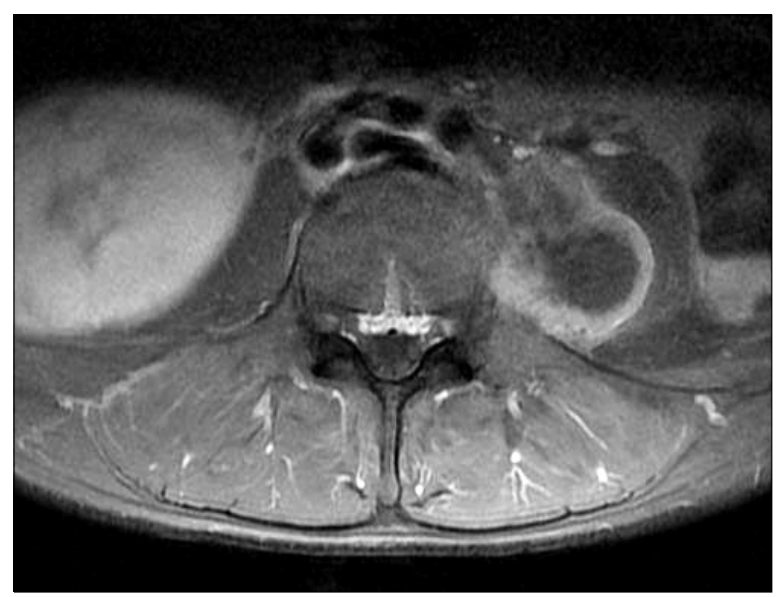

Fig. 2. Axial view (T1 WI) of L-spine MRl shows mass at left iliopsoas muscle. 


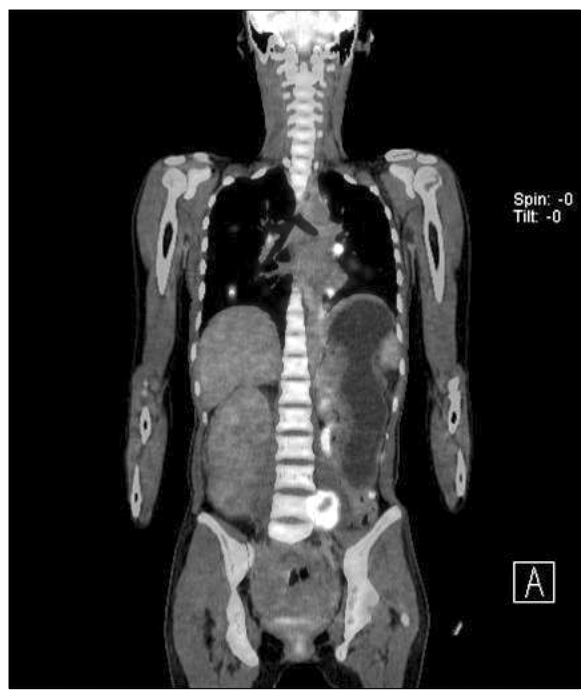

Fig. 3. Coronal view of PET torso shows right suprarenal malignancy with multiple metastases to lungs, pleuras, left psoas muscle and peritoneal seeding.

treated. Her abdominal X-rays revealed an ileus and abdominal computer tomography tests showed a huge retroperitoneal mass. These findings led to performing lumbar spine magnetic resonance imaging (MRI) (Fig. 2), which found disc degeneration accompanied by an annular tear in L5-S1 as well as a mass in her left iliopsoas muscle. In addition, her torso PET scans (Fig. 3) revealed a suprarenal mass in her right side, as well as tumors seeded in her left psoas muscle, in both lungs, and in her pleura and peritoneum.

A needle aspiration biopsy was performed on the mass in her left psoas muscle. The results showed a well-differentiated liposarcoma. With this diagnosis, the patient was transferred to the department of internal medicine at the hospital to receive treatment for her metastatic retroperitoneal liposarcoma.

\section{DISCUSSION}

Isolated femoral neuropathy is rarely reported as the cause of PPP. In most cases, the causes are iatrogenic factors associated with either direct trauma to the nerves, nerve stretching, or nerve compression. With the increasing diversity in the type and extent of surgical procedures, clinicians need to pay more attention to postoperative iatrogenic femoral neuropathy. In fact, postoperative femoral neuropathy has been reported in a number of cases in gynecology, in vascular surgery, and in renal transplantation [3-5].

Since the first study in chronic post-surgical pain was published by Crombie et al. [6] in 1998, a growing interest in PPP has developed among clinicians. PPP is defined as a post-surgical pain that continues for more than two months with no particular reason [2]. Its prevalence rate varies with the types of surgery and each study reports it differently. PPP is clearly a common symptom. According to Kehlet et al. [7], PPP results from persistent nociception or inflammation, or from neuropathic pain due to surgical trauma to the major peripheral nerves.

PPP risk factors include: (a) preoperative factors, such as psychosocial factors; (b) intraoperative factors, such as anesthesia, surgical approaches, and nerve injuries; and (c) postoperative factors such as analgesia use, and earlystage neurophysiological assessment [1]. The most commonly recognized cause of PPP is iatrogenic neuropathic pain. Using a minimally invasive surgical approach is important in the prevention of PPP.

Anatomically speaking, the lumbar plexus is situated anterior to the transverse process of the lumbar vertebrae (L2-5) or in the posterior one third of the psoas major, which is found anterior to the transverse processes of the lumbar vertebrae. Numerous terminal branches arise from the lumbar plexus, some of which originate from the lateral border of the psoas major. The femoral nerve, which is the largest branch of the lumbar plexus and which is situated in the posterior area of the psoas major, lies between the psoas and the iliacus. It exits the pelvis, passes deep to the inguinal ligament; and bifurcates into anterior and posterior divisions [8]. The femoral nerve provides motor innervations to the iliacus and to the pectineus, sartorius, and quadriceps femoris muscles. The nerve also provides sensory innervations to the distal two thirds of the anteromedial aspect of the thigh and to the medial aspect of the leg. An injury to the femoral nerve will lead to numbness and paresthesia in the anterior thigh, the medial calf, medial foot, or the great toe, as well as to pain sensations in the iliac fossa, inguinal region, anterior thigh, or the medial region of the calf. If a case of femoral neuropathy is suspected, the possibility of misdiagnosis must be verified with L4 radiculopathy or lumbar plexopathy to ensure the accuracy of the diagnosis.

In $\mathrm{L} 4$ radiculopathy, dermatomes from the regions of the patient's major complaints are different from those of 
femoral neuropathy. This helps to distinguish the two conditions. Symptoms of L4 radiculopathy include pain in the anterior thigh and medial knee, paresthesia in the anteromedial side of the tibia, weakness in the tibialis anterior, deterioration in the quadriceps femoris muscles and hip adductor, and reduction or loss of patellar tendon reflexes [9]. Lumbar plexopathy symptoms include weakness in the hip flexors and adductor, weakness in the knee extensor, sensory loss in the areas innervated by the femoral, obturator, and lateral femoral cutaneous nerves, and deterioration in the iliopsoas muscle.

In patients diagnosed with femoral neuropathy, the iliopsoas and the hip adductor and flexors must be examined more closely. A clinical diagnosis of iatrogenic femoral neuropathy lesions can be assisted by imaging techniques and electrodiagnostic studies. MRI tests also help with the examination of intraspinal pathology and can help eliminate the possibility of retroperitoneal hematoma. A diagnosis can be confirmed by EMG tests of the denervation and recruitment changes in the iliopsoas, quadriceps and sartorius muscles [10]. Moreover, Henry C. Tong reported using appropriate diagnostic criteria which helped ensure good specificity while examining for lumbosacral radiculopathy [11].

Soft tissue sarcoma is a rare disease. It is a neoplasm arising from the mesenchymal cells and includes adipose, muscle, and connective tissues. Soft tissue sarcoma can be found inside all parts of the body, but they are most frequently found in the proximal area of the lower extremities. With this type of sarcoma, the wide excision of the primary tumor is the key to successful treatments and radiation therapy is required even after complete resection [12]. In patients with soft tissue sarcoma, early diagnosis is often delayed and an aggressive invasion of muscles, bones, and nerves is common. Uncontrollable pain accompanies the condition as it progresses. In comparison, fibromatosis is defined as an infiltrating fibroblastic proliferation, which shows no histological sign of inflammation or clearly distinguished neoplasms. Fibromatosis is difficult to distinguish from low-grade fibrosarcoma, although fibromatosis does not develop into a malignant tumor and is not metastatic to the distal area. These differences help in making an accurate diagnosis. Local recurrence is common in fibromatosis, and, thus, a wide excision of the lesion extending from $2 \mathrm{~cm}$ to $3 \mathrm{~cm}$ from the tumor is the recommended mode of treatment [13].
In our patient, her preoperative pain, limited to the mass in her thigh, persisted after the operation. Moreover, its pathology, intensity, and location continued to change. The pain, sensed only in the mass before the surgery, after surgery started to infiltrate into the areas that are innervated by the femoral nerve. The pain demonstrated neuropathic components and was rapidly aggravated. Based on these findings, we assumed that a peripheral nerve injury resulting from the procedure might have affected the progression of her pain. Furthermore, we suspected, based on our examination of EMG results, postoperative femoral neuropathy that resulted from surgery-induced direct damage to the femoral nerve. Treatments were provided accordingly but were found to have only a limited effect on the pain that continued to affect the patient. Based on her report of feeling localized pain in her left quadratus lumborum and on our observation of limited flexion in her hip, we assumed the likely origin of her pain was the liposarcoma that was found earlier in her lumbar plexus.

In this case study, we ignored the possibility of more serious nerve damage, such as damages to the plexus, and settled on a more fundamental diagnosis of peripheral nerve injury as the cause of the patient's PPP. The recent surge in interest in PPP in the medical community may play a part in clinicians delivering a misdiagnosis due to the lack of a thorough evaluation of the patient's symptoms, while there can be many causes of the pain after the surgery. Our patient experienced what was considered a persistent neuropathic pain after undergoing an excision of a benign tumor in her thigh. Our initial diagnosis was that her pain was caused by postoperative femoral neuropathy, and we provided treatments, accordingly. However, an imaging test revealed a metastatic tumor in her iliopsoas muscle, and our diagnosis eventually changed to tumor induced lumbar plexopathy.

We report this case, along with our review of the literature, to emphasize the importance of performing a more thorough and accurate diagnosis when treating PPP.

\section{REFERENCES}

1. Kehlet H, Rathmell JP. Persistent postsurgical pain: the path forward through better design of clinical studies. Anesthesiology 2010; 112: 514-5.

2. Niraj G, Rowbotham DJ. Persistent postoperative pain: where 
are we now? $\mathrm{Br} J$ Anaesth 2011; 107: 25-9.

3. Pechter EA, Smith PB. Transient femoral neuropathy after abdominoplasty. Ann Plast Surg 2008; 61: 492-3.

4. Van Veer $\mathrm{H}$, Coosemans W, Pirenne J, Monbaliu D. Acute femoral neuropathy: a rare complication after renal transplantation. Transplant Proc 2010; 42: 4384-8.

5. Wilson M, Ramage L, Yoong W, Swinhoe J. Femoral neuropathy after vaginal surgery: a complication of the lithotomy position. J Obstet Gynaecol 2011; 31: 90-1.

6. Crombie $\mathbb{K}$, Davies HT, Macrae WA. Cut and thrust: antecedent surgery and trauma among patients attending a chronic pain clinic. Pain 1998; 76: 167-71.

7. Kehlet $H$, Jensen TS, Woolf CJ. Persistent postsurgical pain: risk factors and prevention. Lancet 2006; 367: 1618-25.

8. Planner AC, Donaghy M, Moore NR. Causes of lumbosacral plexopathy. Clin Radiol 2006; 61: 987-95.
9. Lee GH. Disc herniation. In: Pain medicine. 3rd ed. Edited by The Korean pain society. Seoul, Koonja Publishing Inc. 2007, pp 271-7.

10. Al-Ajmi A, Rousseff RT, Khuraibet AJ. latrogenic femoral neuropathy: two cases and literature update. J Clin Neuromuscul Dis 2010; 12: 66-75.

11. Tong HC. Specificity of needle electromyography for lumbar radiculopathy in 55- to 79-yr-old subjects with low back pain and sciatica without stenosis. Am J Phys Med Rehabil 2011; 90: 233-8.

12. Prendergast B, Fiveash JB, Gibbs CP, Scarborough MT, Indelicato DJ. Radiotherapy for soft tissue sarcoma of the proximal lower extremity. Sarcoma 2010; 2010: 829498.

13. Mackenzie DH. The fibromatoses: a clinicopathological concept. Br Med J 1972; 4: 277-81. 